\title{
A frequency dependent embrittling effect of high pressure hydrogen in a 17-4 PH martensitic stainless steel
}

\author{
Jean-Gabriel Sezgin ${ }^{1, *}$, Junichiro Yamabe ${ }^{1,2,3}$ \\ ${ }^{1}$ AIST-Kyushu University Hydrogen Materials Laboratory (HydroMate), National Institute of Advanced Industrial Science and \\ Technology (AIST), West Zone 1,4F, D-409 Ito Campus, Kyushu University, 744 Moto-oka, Nishi-ku, Fukuoka, 819-0395 Japan \\ ${ }^{2}$ International Research Center for Hydrogen Energy, Kyushu University, 744 Moto-oka, Nishi-ku, Fukuoka, 819-0395 Japan \\ ${ }^{3}$ Research Center for Hydrogen Industrial Use and Storage (HYDROGENIUS), Kyushu University, 744 Moto-oka, Nishi-ku, Fukuoka, \\ 819-0395 Japan
}

\begin{abstract}
The effects of hydrogen on tensile and fatigue-life properties of 17-4PH H1150 steel have been investigated by using a smooth, round-bar specimen for tensile tests and circumferentially-notched specimen for fatigue-life tests. The specimens were precharged by an exposure to $35-100 \mathrm{MPa}$ hydrogen gas at $270^{\circ} \mathrm{C}$ for $200 \mathrm{~h}$. For the $100 \mathrm{MPa}$ hydrogen exposure, the steel showed a significant degradation in ductility loss, translated by a relative reduction in area, RRA, of 0.31 . The fatigue-life test of the present notch specimen (stress concentration factor of 6.6) reflects the fatigue crack growth (FCG) for long cracks. The fatigue limit of the non-charged and H-charged notched specimens, defined by the threshold of non-propagation for long cracks, was not affected by hydrogen. At a higher stress amplitude, the H-charged specimen showed a significant FCG acceleration ratio compared to the non-charged specimen. Although, an upper bound of the FCG acceleration seemed to exist, this ratio was approximately 100 . The fracture surface of the H-charged specimen was covered with quasi-cleavage (QC) at a lower stress amplitude and with a mixture of QC and intergranular (IG) facets at higher stress amplitudes. It has been suggested that a cycle-dependent crack growth accompanied by QC occurs at a lower stress amplitude, whereas a mixture of cycle-dependent crack growth (accompanied by QC) and time-dependent crack growth (accompanied by IG) occurs otherwise. This mixture justifies the 100 times FCG acceleration ratio in spite of the existence of the upper bound.
\end{abstract}

\section{Introduction}

Hydrogen has been recently considered to be a sustainable carrier in order to tackle the future energetic challenges. However, a limited number of steels is available to manufacture such systems due to hydrogen compatibility issues. To enhance cost-effectiveness (e.g. of FCV), substantial efforts have been assigned to clarify the degradation of mechanical and metallurgical properties directly caused by hydrogen. This phenomenon is usually called Hydrogen Embrittlement (HE) [1]-[3]. Depending on the supply of hydrogen, the application and the nature of the alloy, various mechanisms are available in the literature [4]-[8], however, this phenomenon has not been deeply understood and a precise mechanism has not been established yet.

From the viewpoint of strength design for components, the utilization of high-strength steels is relevant to combine weight reduction and high performance. However, extensive experimental results demonstrate that the high-strength steel with ultimate tensile strength (UTS) exceeding approximately $1 \mathrm{GPa}$ is considerably or severely susceptible to HE [9]-[18]. In addition, it has also been reported that an upper bound of fatigue crack growth (FCG) ratio by hydrogen (30 times acceleration) exists only for low- or medium-strength steels with UTS $<900 \mathrm{MPa}$ [18]. This fact suggests that the HE mechanism of high-strength steels may be different from the mechanism observed on low- and medium-strength ones. Data available for some steels such as JIS-SCM435 and JIS-SNCM439 with UTS $<900 \mathrm{MPa}$ [18] or the SAE52100 (JIS-SUJ2) with UTS of $1900 \mathrm{MPa}$ [19] support this assumption. Fig. 1 shows a schematic illustration of the test frequency dependence of the hydrogen-enhanced FCG acceleration ratio of these steels. For the low-alloy steels with UTS $<900 \mathrm{MPa}$, an upper bound of the hydrogenenhanced FCG acceleration ratio exists and the fatigue crack presents a cycle-dependent growth. This fracture surface is covered with quasi-cleavage (QC) and the acceleration is explained by the hydrogen-induced successive crack growth (HISCG) model [20] based on localized slip deformations enhanced by hydrogen. In contrast, for the high-strength steel with a UTS of 1.9 $\mathrm{GPa}$, no upper bound of the hydrogen-enhanced FCG acceleration ratio exists and this fatigue crack shows a time-dependent growth. This fracture surface is covered with intergranular (IG) surface and the acceleration is explained by the hydrogen-enhanced deformation twin model [19].

In the current paper, the material of interest is a 17$4 \mathrm{PH}$ stainless steel with an UTS of around $1 \mathrm{GPa}$. It is well-known that the 17-4PH steel is susceptible to HE [21]. The objective of this paper is to clarify the effect of hydrogen on the threshold behaviour for long crack, the FCG acceleration of the steel and then, propose an appropriate mechanism to explain the hydrogenenhanced FCG acceleration.

"Corresponding author: sezgin.jean-gabriel@aist.go.jp 


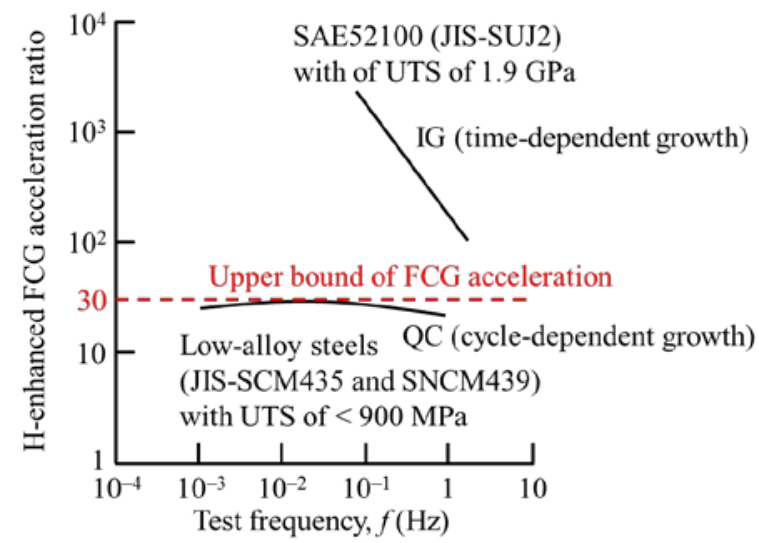

Fig. 1. Test frequency dependence of hydrogen-enhanced FCG acceleration for various steels [18], [19]

\section{Material characterization}

\subsection{Metallurgical aspects}

The material used in this paper is a $17-4 \mathrm{PH}$ stainless steel. The chemical composition is given in Table 1. To adjust its mechanical properties, the steel is heat-treated in two steps. The first step is a solution treatment at $1040^{\circ} \mathrm{C}$ for $1 \mathrm{~h}$ with water quenching. The second step is a precipitation hardening treatment at $620^{\circ} \mathrm{C}$ for $2 \mathrm{~h}$ with air cooling. This heat-treated steel is referred to as a 174PH H1150 steel.

Table 1. Chemical composition of the 17-4 PH steel in mass $\%$

\begin{tabular}{|c|c|c|c|c|c|}
\hline & $\mathbf{C}$ & $\mathbf{S i}$ & $\mathbf{M n}$ & $\mathbf{P}$ & $\mathbf{S}$ \\
\hline \multirow{3}{*}{$\mathbf{1 7 - 4} \mathbf{~ P H}$} & 0.04 & 0.31 & 0.87 & 0.034 & 0.004 \\
\cline { 2 - 5 } & $\mathbf{C u}$ & $\mathbf{N i}$ & $\mathbf{C r}$ & $\mathbf{N b}$ & \\
\cline { 2 - 5 } & 3.3 & 4.24 & 15.57 & 0.34 & \\
\hline
\end{tabular}

After heat treatment, the obtained microstructure is presented on Fig. 2-a). This SEM micrograph is obtained by using the backscattered electron (BSE) signals and suggests a martensitic microstructure. This point is confirmed by the electron backscatter diffraction (EBSD) map given in Fig. 2-b). This inverse pole figure (IPF) map clearly shows the characteristic lath of a martensitic microstructure. During martensitic transformation, in case of a high cooling rate, some residual austenite may remain in the alloy, influencing therefore the repartition of hydrogen in solution. The proportion of residual austenite in martensitic structures is quite important for two reasons. The first one is the high content of hydrogen in these areas because of the high solubility. The second one is the metastability of this phase. The phase map in Fig. 2-c) shows the proportion of FCC iron (in red) contained in the BCC structure (in green). The proportion of residual austenite has been measured and is below $4 \%$. This fact disproves any hypotheses relying on an effect of strain induced reversion of martensite.

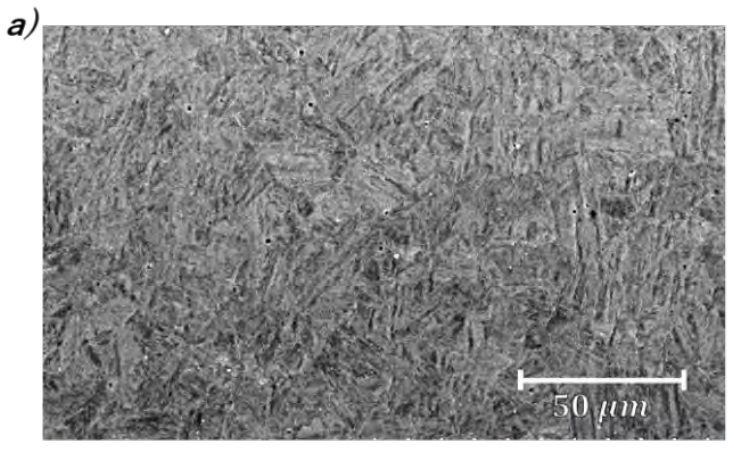

b)

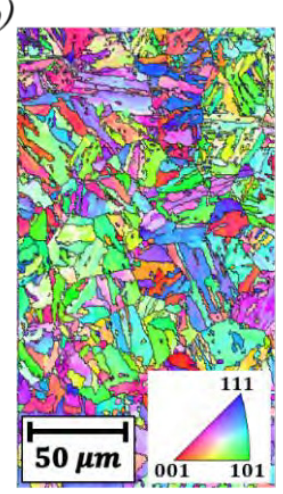

c)

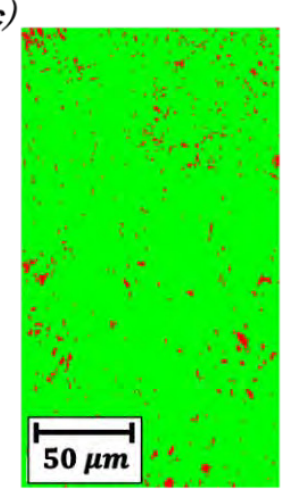

Fig. 2. Microstructure of 17-4PH H1150: (a) BSE image, (b) IPF map, and (c) phase map. Maps of (b) and (c) were obtained by EBSD

\subsection{Sample preparation and test procedures}

The three types of specimen used in this study are illustrated in Fig. 3. The first geometry used for tensile tests is a smooth, round-bar specimen, as shown on Fig. 3-a). The tensile tests of non-charged and $\mathrm{H}$-charged specimens were performed in air at room temperature at a displacement rate of $1 \mathrm{~mm} / \mathrm{min}$.

The fatigue-life tests were performed on a circumferentially-notched specimen as shown in Fig. 3b). The specimen has been designed in accordance with the ASTM G142-98 standard [22]. The net section diameter is $6.4 \mathrm{~mm}$ at notch root with a nominal root radius specified at $0.083 \mathrm{~mm}$, while the gross section diameter is $12.8 \mathrm{~mm}$. The stress concentration factor $\left(K_{\mathrm{t}}\right)$ for this geometry is about 6.6. The tests of non-charged and $\mathrm{H}$-charged specimens were conducted in air at room temperature at a stress ratio, $R$, of -1 and under test frequencies ranging from 0.001 to $1 \mathrm{~Hz}$. The existing literature [23] has demonstrated that the fatigue life of this notched specimen can be predicted from FCG rate obtained with an ordinary compact-tension (CT) specimen. Thus, the fatigue-life test of the present notched specimen evaluates the FCG behavior of 17 4PH H1150.

After fatigue-life tests, the hydrogen content is measured from the specimens described in Fig. 3-c). These specimens are processed from fatigue-life ones. 
a)

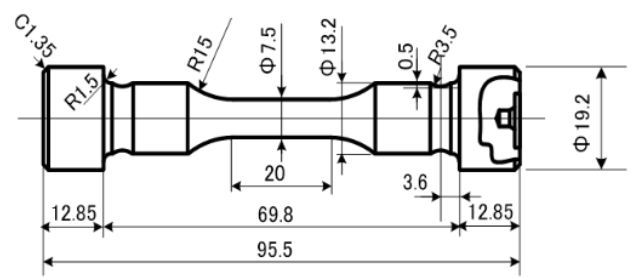

b)

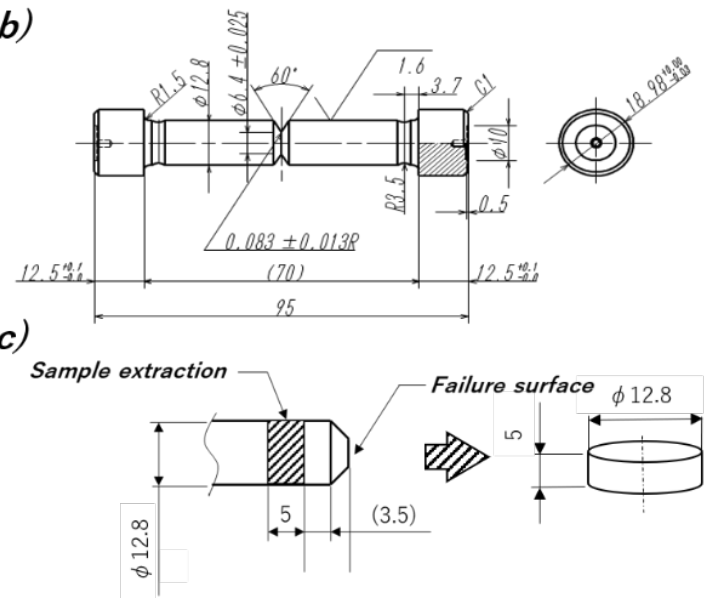

Fig. 3. Specimen geometries used in (a) tensile tests, (b) in fatigue-life tests and (c) in hydrogen-content measurements

\subsection{Hydrogen charging and hydrogen-content measurements}

Before the tensile and fatigue-life tests, some specimens were exposed to $35 \mathrm{MPa}$ or $100 \mathrm{MPa}$ hydrogen gas at $270^{\circ} \mathrm{C}$ for $200 \mathrm{~h}$. According to previous investigations, the present exposure condition ensures the uniform distribution of hydrogen content in the specimen. During the fatigue-life tests, hydrogen desorption from the specimens may occur; thus, the hydrogen content after fatigue-life tests were measured using thermal desorption analysis (TDA) under a rising temperature at a heating rate of $100^{\circ} \mathrm{C} / \mathrm{h}$. The samples used for the TDA measurements were extracted far enough from the failure area, at $5 \mathrm{~mm}$ of the net section, and have a diameter of $12.8 \mathrm{~mm}$ and a height of $5 \mathrm{~mm}$ (Fig. 3-c).

\section{Tensile properties}

The nominal stress-strain curves are provided in Fig. 4 , and show that barely no effects of hydrogen on the elastic behavior as well as the UTS are observable. The values of UTS were $1013 \mathrm{MPa}$ (non-charged), $1016 \mathrm{MPa}$ (35 MPa H-charged), and $1026 \mathrm{MPa}$ (100 MPa Hcharged). In contrast, the plastic behavior was strongly affected by hydrogen. The elongation at failure of the non-charged specimen was $37 \%$ and the ratio in elongation (compared to the non-charged specimen was 0.43 for the $35 \mathrm{MPa} \mathrm{H}$-charged specimen and 0.38 for the $100 \mathrm{MPa} \mathrm{H}$-charged specimen. In addition, the ductility loss is usually quantified in terms of relative reduction in area (RRA). The values of RRA were 0.43 for $35 \mathrm{MPa} \mathrm{H}$-charged specimen and 0.31 for $100 \mathrm{MPa}$ $\mathrm{H}$-charged specimen, respectively. From a regulatory point of view [24], this material is therefore strongly affected by hydrogen.

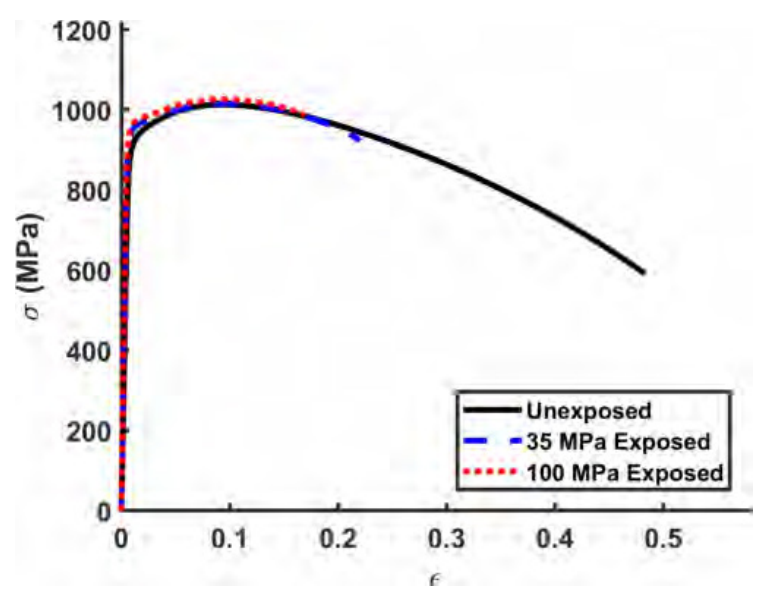

Fig. 4. Nominal stress-strain curves obtained from tensile tests of the non-charged specimen (reference) and H-charged specimens

Fig. 5 shows the fracture surfaces of the tensile specimens. The non-charged specimens failed by an ordinary cup-and-cone ductile failure. In contrast, the $\mathrm{H}$ charged specimen showed a brittle failure, accompanied by a mixture of QC and IG surfaces.

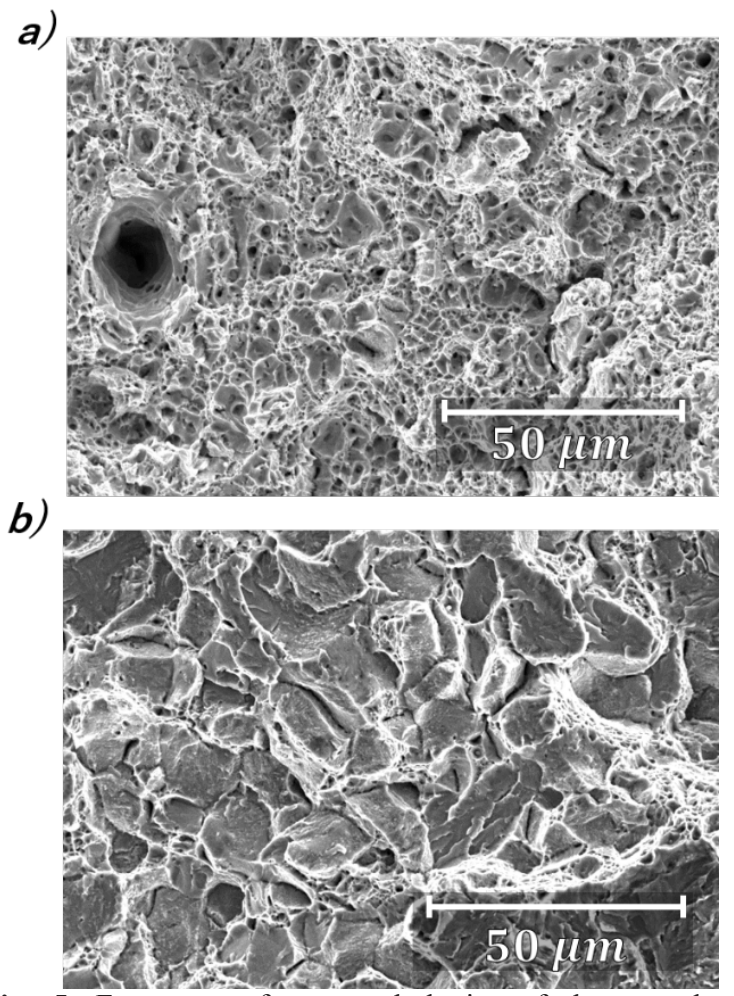

Fig. 5. Fracture surface morphologies of the non-charged tensile specimen (a) and the H-charged tensile specimen (b) by SEM

\section{Fatigue-life properties}

\subsection{Residual hydrogen content}

Because solute hydrogen strongly affects mechanical properties of a material, it is then worthy to measure the hydrogen content in the specimen. Fig. 6 
presents the residual hydrogen content of the H-charged specimens after the fatigue-life tests in air at room temperature. As a reference, residual hydrogen content of the specimens exposed to $10 \mathrm{MPa}$ and $35 \mathrm{MPa}$ hydrogen gas at $270^{\circ} \mathrm{C}$ are also plotted. Regardless the hydrogen-gas pressures, the hydrogen desorption from the H-charged specimens is hardly observable. In addition, the hydrogen content, $C_{\mathrm{H}}$, is higher in case of high pressure of hydrogen gas since the content follows the Sieverts law, i.e., $C_{\mathrm{H}}=S F^{1 / 2}$, where $S$ is the solubility of hydrogen and $F$ is the fugacity.

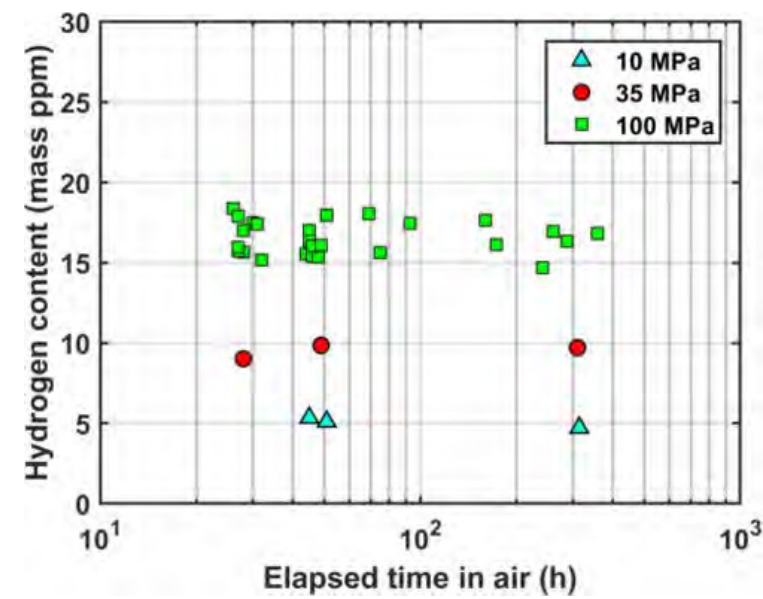

Fig. 6. Residual hydrogen content of H-charged specimens after fatigue-life tests

\section{$4.2 S-N$ and threshold properties}

Fig. 7 shows the $S$ - $N$ curves of the non-charged and $\mathrm{H}$-charged specimens tested in air at room temperature under $R=-1$ and $f=0.01-10 \mathrm{~Hz}$. The data related to the non-charged specimens are represented in light blue, whereas the data referring to the $\mathrm{H}$-charged specimens are represented in red. The fatigue-life of the H-charged specimens is remarkably degraded compared to the noncharged ones in the low-cycle regime. In parallel, within the high-cycle regime, the fatigue-life reduction of the $\mathrm{H}$-charged specimen is limited. In addition, the fatigue limit $\sigma_{w}$, herein defined as the non-failure maximal stress amplitude for $1 \times 10^{7}$ cycles, is equal in case of non-charged and $\mathrm{H}$-charged specimens. This value is $\sigma_{w}=110 \mathrm{MPa}$.

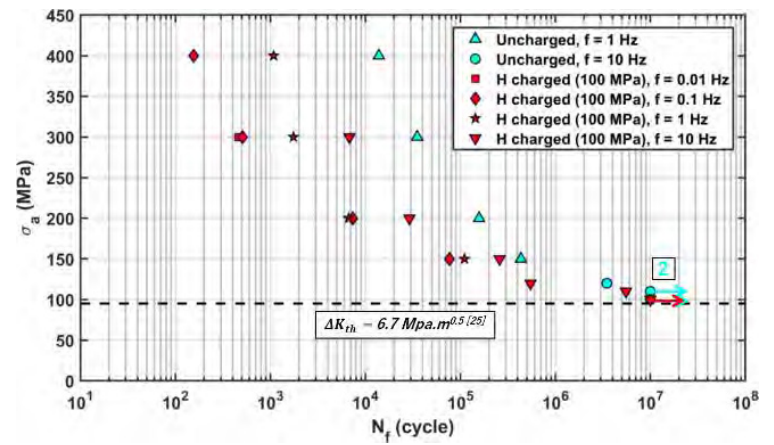

Fig. 7. $S-N$ curves of non-charged and $\mathrm{H}$-charged circumferentially-notched specimens tested in air at room temperature under $\mathrm{R}=-1$ and various test frequencies
Fig. 8 presents optical micrographs of the cross section at the fatigue limit for the non-charged and the $\mathrm{H}$-charged specimens. Fatigue cracks emanating from the notch root are observed on both specimens. However, these cracks are not observable on the non-failed specimens tested at a stress amplitude of $105 \mathrm{MPa}$. Although further investigations are needed, these cracks seem to be non-propagating cracks. The fatigue limit of the circumferentially-notched specimens is therefore determined by the threshold of non-propagation of fatigue cracks (different from the threshold for crack initiation). The dashed black line in Fig. 7 represents the predicted fatigue limit based on the threshold stress intensity factor range for long cracks at $R=-1, \Delta K_{t h}$ found to be $6.7 \mathrm{MPa}^{1 / 2}$ for $17-4 \mathrm{PH}$ steels [25]. To calculate the corresponding stress amplitude, the expression (1) of the stress intensity factor in mode I has been used:

$$
\begin{gathered}
\Delta K_{I}=\Delta \sigma \sqrt{\pi b} F(\xi)=2 \sigma_{a} \sqrt{\pi b} F(\xi) \\
F(\xi)=\frac{1}{2}\left(1+\frac{1}{2} \xi+\frac{3}{8} \xi^{2}-0.363 \xi^{3}+0.731 \xi^{4}\right) \sqrt{1-\xi}
\end{gathered}
$$

where $\xi$ is the ratio of the net diameter, $b$, to the gross diameter, $R$. Although the heat-treatment conditions differ from the literature, the experimental fatigue limit of the circumferentially-notched specimen is reasonably consistent with the predicted fatigue limit based on the threshold value for long crack.
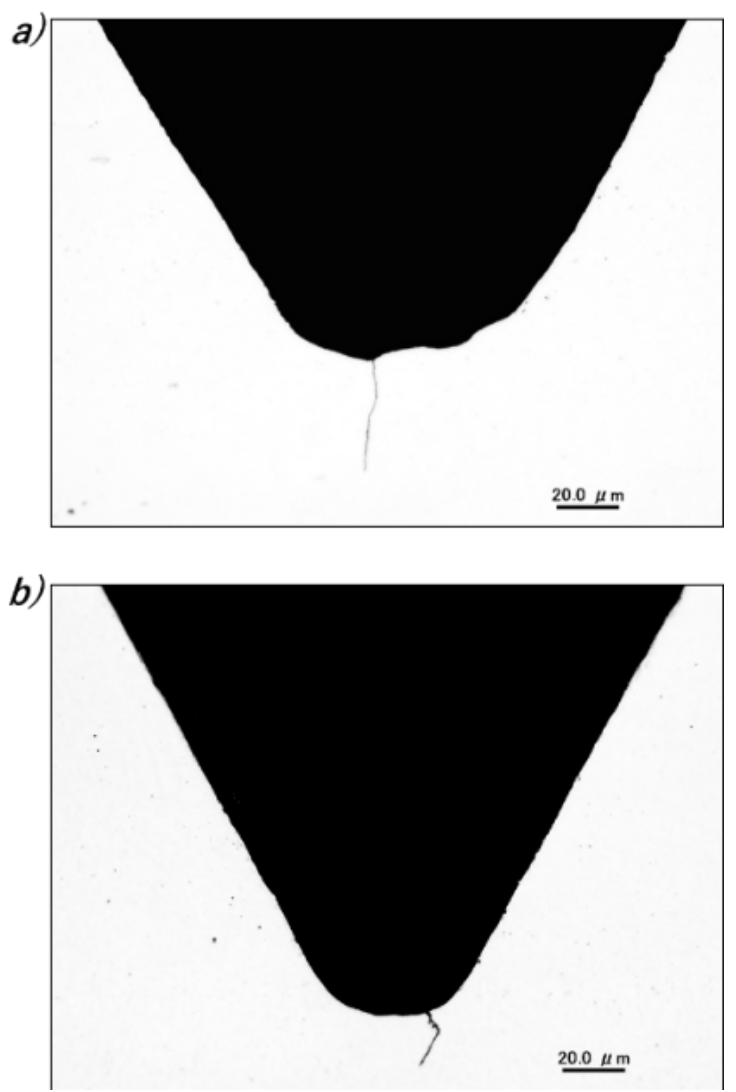

Fig. 8. Non-propagating cracks emanating from the notch root of non-charged and H-charged specimens at the fatigue-limit 
condition. The stress amplitude of both specimens is equal $\left(\sigma_{\mathrm{a}}=110 \mathrm{MPa}\right)$

\subsection{FCG acceleration under various combination of stress amplitudes and test frequencies}

The experimental result in Fig. 7 shows a strong decrease of the fatigue life due to the presence of hydrogen. This decrease is about one order of magnitude depending on the testing frequency. This graph also shows that the embrittling effect of hydrogen is emphasized for lower frequencies and high amplitudes. In contrast, for lower amplitude, the effect of hydrogen is minimized. In order to quantify the impact of hydrogen on the fatigue life, the ratio of the fatigue-life for the non-charged specimen to the $\mathrm{H}$-charged specimen, $N_{f} / N_{f_{H}}$, has been introduced. This parameter represents the ratio of the hydrogen-enhanced FCG acceleration; therefore, $N_{f} / N_{f_{H}}$ is hereinafter referred to as the FCG acceleration ratio.

Fig. 9 presents the evolution of the FCG acceleration ratio as a function of the test frequency for several stress amplitudes (stress ratio $R=-1$ ). Except for the stress amplitude of $400 \mathrm{MPa}$, an upper bound of the FCG acceleration seems to exist, although the acceleration ratio is quantitatively different. The FCG acceleration is promoted at a higher stress amplitude, and its value is approximately 100 times higher in case of $\sigma_{a}=300 \mathrm{MPa}$. As shown in Fig. 1, the hydrogenenhanced FCG acceleration ratio based on the HISCG model is below 30 . Thus, another mechanism, different from the HISCG model, may contribute to the observed FCG acceleration ratio of 100 , although an upper bound of the FCG acceleration seems to exist.

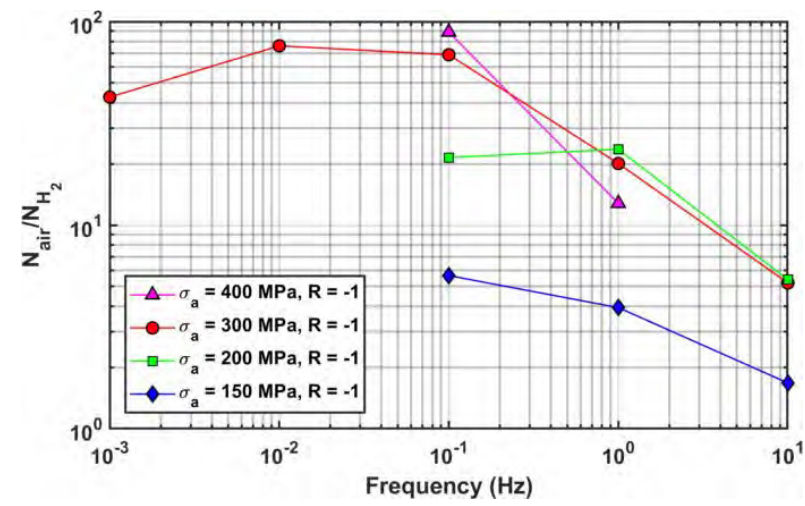

Fig. 9. Ratio of hydrogen-enhanced FCG acceleration under various combinations of stress amplitudes and test frequencies

\subsection{Fracture surface morphologies}

Fracture surface morphologies of non-charged and $\mathrm{H}$-charged specimens have been investigated by SEM imagery. Fig. 10 shows the fracture surface of the noncharged specimen at $\sigma_{a}=120 \mathrm{MPa}$ and $f=10 \mathrm{~Hz}$ (highcycle regime), whereas Fig. 11 shows the fracture surface obtained at $\sigma_{a}=400 \mathrm{MPa}$ at $f=1 \mathrm{~Hz}$ (low-cycle regime). Both surfaces indicate typical fatigue striations or microstructure-dependent surface.

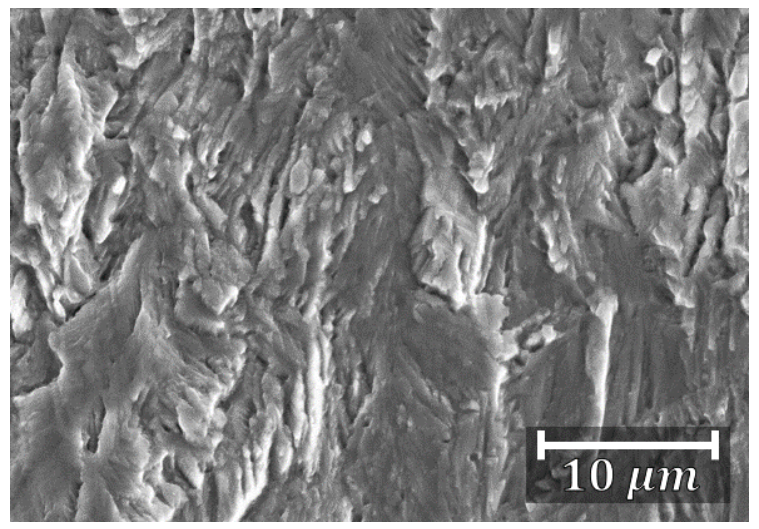

Fig. 10. Fracture surface morphology of the non-charged specimen at $\sigma_{\mathrm{a}}=120 \mathrm{MPa}$ and $f=10 \mathrm{~Hz}$

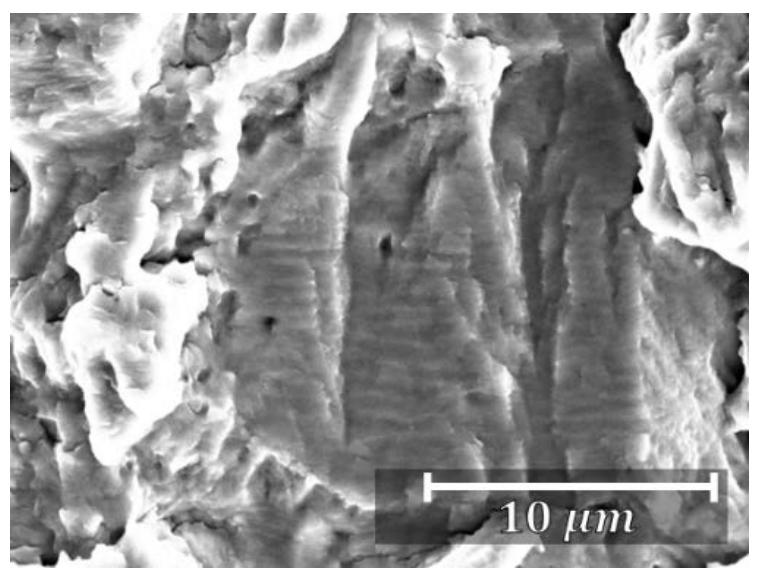

Fig. 11. Fracture surface morphology of the non-charged specimen at $\sigma_{\mathrm{a}}=400 \mathrm{MPa}$ and $f=1 \mathrm{~Hz}$

Fig. 12 presents the fracture surface of the $\mathrm{H}$ charged specimen tested at $\sigma_{a}=120 \mathrm{MPa}$ and $f=10 \mathrm{~Hz}$. The fracture surface is different from the non-charged specimen and is covered with QC surface. The SEM micrograph presented in Fig. 13 is related to the Hcharged specimen tested at $\sigma_{a}=400 \mathrm{MPa}$ and $f=1 \mathrm{~Hz}$. For this condition, the fracture surface is covered with QC and IG surfaces. Fig. 14 also presents the fracture surface of the H-charged specimen at $\sigma_{a}=300 \mathrm{MPa}$ and $f=0.01 \mathrm{~Hz}$. In this case, the FCG acceleration of the Hcharged specimen is approximately 100 times higher compared to the non-charged specimen. The corresponding SEM micrograph reveals both QC and IG surfaces.

Fractographic observations have shown that the non-charged specimens usually fail by fatigue striations. In contrast, the introduction of hydrogen has led to a brittle fracture accompanied by QC and IG surfaces. At higher stress amplitudes, the fracture surface is covered with QC and IG surfaces, whereas at lower stress amplitude, the fracture surface is only covered with QC surface. 


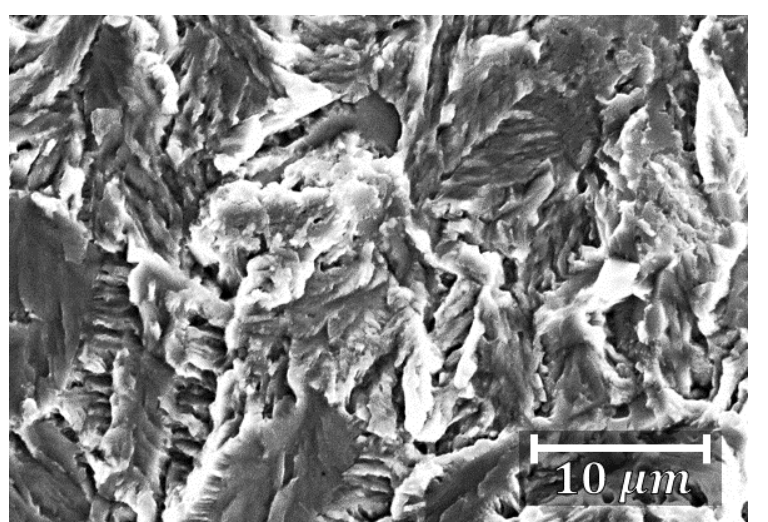

Fig. 12. Fracture surface morphology of the H-charged specimen at $\sigma_{\mathrm{a}}=120 \mathrm{MPa}$ and $f=10 \mathrm{~Hz}$

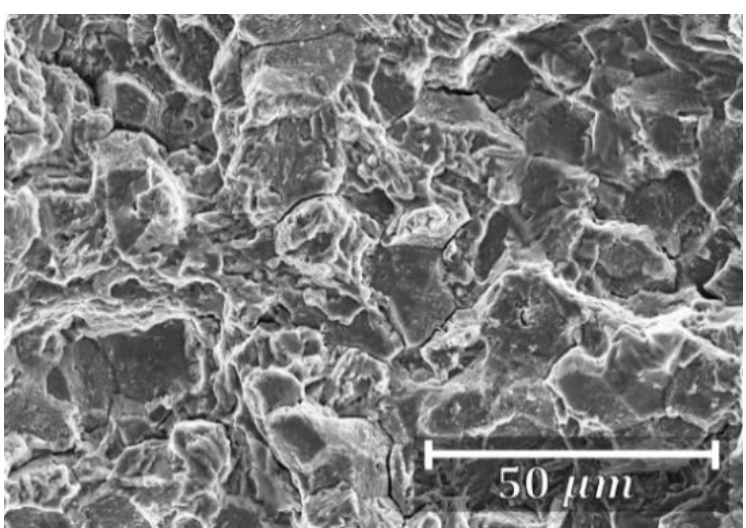

Fig. 13. Fracture surface morphology of the H-charged specimen at $\sigma_{\mathrm{a}}=400 \mathrm{MPa}$ and $f=1 \mathrm{~Hz}$

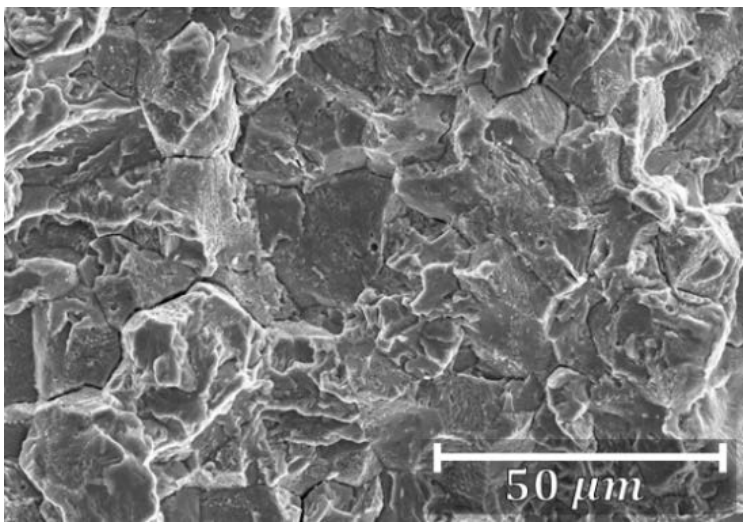

Fig. 14 Fracture surface morphology of the H-charged specimen at $\sigma_{\mathrm{a}}=300 \mathrm{MPa}$ and $f=0.01 \mathrm{~Hz}$

\section{Discussion}

As shown in Fig. 9, the FCG acceleration ratio at a higher stress amplitude is about 100, and an upper bound for the FCG acceleration ratio seems to exist. According to the HISCG model, the fatigue crack growth of the $\mathrm{H}$ charged specimen is at most 30. Thus, another mechanism, different from the HISCG model, may contribute to reach the experimental FCG acceleration ratio of 100 .

For a lower stress amplitude, the FCG acceleration ratio of the $\mathrm{H}$-charged specimens has an upper bound and the actual value is lower than 10. Furthermore, as shown in Fig. 12, the fracture surface was only covered with QC surface. In consequence, it is considered that such failure surface results from the HISCG model.

In contrast, for a higher stress amplitude, as known in Fig. 13 and Fig. 14, the fracture surface was covered with QC and IG surfaces. It means that both timedependent crack growth (leading to IG areas) and cycledependent crack growth (leading to QC areas, result of the HISCG mechanism) occur. It is therefore proposed that the resulting mechanism is issued from the superposition of both elementary mechanisms as expressed in (2).

$$
\frac{N_{f}}{N_{f_{H}}}=\alpha\left(\frac{N_{f}}{N_{f_{H}}}\right)_{Q C}+(1-\alpha)\left(\frac{N_{f}}{N_{f_{H}}}\right)_{I G}
$$

where $\left(N_{f} / N_{f_{H}}\right)$ is the acceleration ratio considering only the mechanism $i$ and $\alpha$ is the surface fraction which have failed by QC. Fig. 15 shows a schematic illustration of the frequency dependence of FCG acceleration ratio of H-charged specimens at higher and lower stress amplitudes. In case of both QC and IG failure, the resulting FCG acceleration ratio of the $\mathrm{H}$-charged specimens appears to result from the superposition of a cycle-dependent crack growth (based on the HISCG mechanism) and a time-dependent crack growth. Consequently, a larger surface fraction of IG leads to an enhanced contribution of the time-dependent crack growth.

In the current study, the fraction of IG area represents approximately $20 \%$ of the failure surface for the $\sigma_{a}=300 \mathrm{MPa}$ condition (measurement performed at on a larger surface than Fig. 14). Therefore, the contribution of the cycle-dependent crack growth is higher than the time-dependent crack growth. As a result, it is considered that at a higher stress amplitude, the $\mathrm{H}$ charged specimens present an upper bound for the FCG acceleration ratio. However, this ratio is approximately 100 , which is considerably higher than the ratio expected based on the HISCG $(\sim 30)$. Similar tendencies have been observed on low-alloy steels (JIS-SCM435 and JISSNCM439) with UTS > $900 \mathrm{MPa}$ [18].

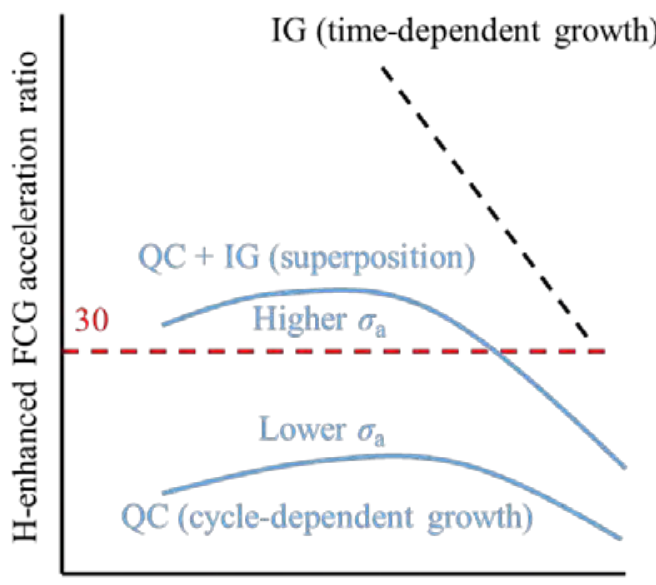

Test frequency 
Fig. 15. Schematic illustration of test frequency dependence of FCG acceleration ratio of the H-charged specimens at higher and lower stress amplitudes

\section{Conclusion}

This paper has investigated the effect of hydrogen on threshold and fatigue crack growth (FCG) properties of the 17-4PH H1150 stainless steel under a wide range of test frequencies. Smooth round-bar specimens for tensile tests and circumferentially-notched specimens for fatigue-life tests have been used and exposed to $100 \mathrm{MPa}$ hydrogen gas at $270^{\circ} \mathrm{C}$ for $200 \mathrm{~h}$. The tensile and fatigue-life tests of non-charged and $\mathrm{H}$-charged specimens have been performed in air at room temperature. The following points have been put in evidence:

1. This steel is highly sensitive to hydrogen since the relative reduction in area (RRA) was found to be 0.43 and 0.31 in respect with the $35 \mathrm{MPa}$ and $100 \mathrm{MPa} \mathrm{H}$-charging conditions (prior to the tensile tests).

2. For H-charged specimens, hydrogen has not significantly desorbed during the fatigue-life tests and is almost constant.

3. The fatigue limit of the circumferentially-notched specimens has been determined considering the threshold for non-propagation of fatigue cracks. The tests on the non-charged and H-charged specimen have revealed that the fatigue limit is not affected by hydrogen.

4. At higher stress amplitudes, the H-charged specimen presented a significant FCG acceleration ratio compared to the non-charged specimen. Although an upper bound of the hydrogenenhanced FCG acceleration ratio seems to exist, this ratio was approximately 100 . In contrast, the hydrogen-enhanced FCG acceleration ratio at lower stress amplitudes is lower than 10.

5. The H-charged specimens showed a fracture surface covered with quasi-cleavage (QC) at lower stress amplitudes and a mixture of QC and intergranular (IG) at higher stress amplitudes.

6. It has been suggested that the crack growth is cycle-dependent (accompanied by QC) at a lower stress amplitude. Otherwise, a mixture of cycledependent crack growth (QC) and time-dependent crack growth (accompanied by IG areas) seems to take place.

\section{References}

[1] A. M. Brass and J. Chene, "Influence of deformation on the hydrogen behavior in iron and nickel base alloys: A review of experimental data," Mater. Sci. Engng A, vol. 242, pp. 210221, 1998.

[2] J. P. Hirth, "Effects of hydrogen on the properties of iron and steel," Metall. Trans. A, vol. 11, no. 6, pp. 861-890, 1980.

[3] R. P. Gangloff and B. P. Somerday, Gaseous hydrogen embrittlement of materials in energy technologies: mechanisms, modelling and future developments. Elsevier, 2012.

[4] L. Coudreuse, Fragilisation par l'hydrogène et corrosion sous contrainte; Corrosion sous contrainte - phénoménologie et mécanismes, Ed. D. Des. 1992.

[5] Q. Liu, Q. Zhou, J. Venezuela, M. Zhang, J. Wang, and A. Atrens, "A review of the influence of hydrogen on the mechanical properties of DP, TRIP, and TWIP advanced high-strength steels for auto construction," Corros. Rev., vol. 34, no. 3, pp. 127-152, 2016.

[6] Q. Liu and A. Atrens, "A critical review of the influence of hydrogen on the mechanical properties of medium-strength steels," Corros. Rev., vol. 31, no. 3-6, pp. 85-103, 2013.

[7] J. Venezuela, Q. Liu, M. Zhang, Q. Zhou, and A. Atrens, "A review of hydrogen embrittlement of martensitic advanced high-strength steels," Corros. Rev., vol. 34, no. 3, pp. 153-186, 2016.

[8] S. Ramamurthy and A. Atrens, "Stress corrosion cracking of high-strength steels," Corros. Rev., vol. 31, no. 1, pp. 1-31, 2013.

[9] R. J. Walter and W. T. Chandler, "Effects of high-pressure hydrogen on storage vessel materials," 1968.

[10] G. Sandoz, "A unified theory for some effects of hydrogen source, alloying'elements, and potential on crack growth in martensitic AISI 4340 steel," Metall. Trans., vol. 3, no. May, pp. 1169-1176, 1972.

[11] W. T. Chandler and R. J. Walter, "Testing to determine the effect of high-pressure hydrogen environments on the mechanical properties of metals," in Hydrogen embrittlement testing, ASTM International, 1974.

[12] W. T. Chandler and R. J. Walter, "Hydrogenenvironment embrittlement of metals and its control," 1975.

[13] J. Toplosky and R. O. Ritchie, "On the influence of gaseous hydrogen in decelerating fatigue crack growth rates in ultrahigh strength steels," Scr. Metall., vol. 15, no. 8, pp. 905-908, 1981.

[14] N. Bandyopadhyay, J. Kameda, and C. J. Mcmahon, "Hydrogen-induced cracking in 4340-type steel: Effects of composition, yield strength, and H2 pressure," Metall. Trans. A, vol. 14, no. 4, pp. 881-888, 1983.

[15] R. P. Gangloff, "Hydrogen assisted cracking of high strength alloys," Compr. Struct. Integr., vol. 6, pp. 31-101, 2003.

[16] C. San Marchi and B. P. Somerday, "Technical Reference On Hydrogen Compatibility Of Materials," Sand2008-1163, no. code 4001, p. 292, 2012.

[17] J. Yamabe, H. Itoga, T. Awane, T. Matsuo, H. Matsunaga, and S. Matsuoka, "Pressure Cycle Testing of $\mathrm{Cr}$ - Mo Steel Pressure Vessels Subjected to Gaseous Hydrogen," vol. 138, no. February, pp. 1-13, 2016.

[18] S. Matsuoka, H. Matsunaga, J. Yamabe, S. Hamada, and T. Iijima, "Various strength 
properties of SCM435 and SNCM439 low-alloy steels in $115 \mathrm{MPa}$ hydrogen gas and proposal of design guideline," Trans. JSME (in Japanese), vol. 83, no. 854, pp. 17-264-17-00264, 2017.

[19] J. Yamabe, T. Matsumoto, S. Matsuoka, and Y. Murakami, "A new mechanism in hydrogenenhanced fatigue crack growth behavior of a 1900-MPa-class high-strength steel," Int. J. Fract., vol. 177, no. 2, pp. 141-162, 2012.

[20] S. Matsuoka, J. Yamabe, and H. Matsuoka, "Criterial for determinating hydrogen compatiblity and the mechanisms for hydrogenassisted, surface crack growth in austenitic stainless steels," Eng. Fract. Mech., vol. 153, no. 3, pp. 103-127, 2016.

[21] L. W. Tsay, W. C. Lee, R. K. Shiue, and J. K. $\mathrm{Wu}$, "Notch tensile properties of laser-surfaceannealed 17-4 PH stainless steel in hydrogenrelated environments," Corros. Sci., vol. 44, no. 9, pp. 2101-2118, 2002.

[22] A. Standard, "G142-98" Standard test method for determination of susceptibility of metals to embrittlement in hydrogen containing environments at high pressure, high temperature, or both," ASTM Int. West Conshohocken, PA, vol. 4, 2004.

[23] J. Yamabe et al., "Qualification of chromiummolybdenum steel based on the safety factor multiplier method in CHMC1-2014," Int. J. Hydrogen Energy, vol. 40, no. 1, pp. 719-728, 2015.

[24] “ANSI/CSA CHMC 1 - 2014: Test Method for Evaluating Material Compatibility in Compressed Hydrogen Applications - Phase I Metals," CSA Gr., 2014.

[25] B. M. Schönbauer, K. Yanase, and M. Endo, "The influence of various types of small defects on the fatigue limit of precipitation-hardened 174PH stainless steel," Theor. Appl. Fract. Mech., vol. 87, pp. 35-49, 2017. 